\title{
Nucleus Accumbens Acetylcholine Receptors Modulate Dopamine and Motivation
}

\author{
Anne L Collins', Tara J Aitken', Venuz Y Greenfield', Sean B Ostlund ${ }^{2}$ and Kate M Wassum ${ }^{*, 1,3}$ \\ 'Department of Psychology, UCLA, Los Angeles, CA, USA; '2Department of Anesthesiology and Perioperative Care, UCI, Irvine, CA, USA; ${ }^{3}$ Brain \\ Research Institute, UCLA, Los Angeles, CA, USA
}

\begin{abstract}
Environmental reward-predictive cues can motivate reward-seeking behaviors. Although this influence is normally adaptive, it can become maladaptive in disordered states, such as addiction. Dopamine release in the nucleus accumbens core (NAc) is known to mediate the motivational impact of reward-predictive cues, but little is known about how other neuromodulatory systems contribute to cue-motivated behavior. Here, we examined the role of the NAc cholinergic receptor system in cue-motivated behavior using a Pavlovian-to-instrumental transfer task designed to assess the motivating influence of a reward-predictive cue over an independently-trained instrumental action. Disruption of NAc muscarinic acetylcholine receptor activity attenuated, whereas blockade of nicotinic receptors augmented cue-induced invigoration of reward seeking. We next examined a potential dopaminergic mechanism for this behavioral effect by combining fast-scan cyclic voltammetry with local pharmacological acetylcholine receptor manipulation. The data show evidence of opposing modulation of cue-evoked dopamine release, with muscarinic and nicotinic receptor antagonists causing suppression and augmentation, respectively, consistent with the behavioral effects of these manipulations. In addition to demonstrating cholinergic modulation of naturally-evoked and behaviorally-relevant dopamine signaling, these data suggest that NAc cholinergic receptors may gate the expression of cue-motivated behavior through modulation of phasic dopamine release.

Neuropsychopharmacology (2016) 4I, 2830-2838; doi:I0.1038/npp.2016.8I; published online 10 August 2016
\end{abstract}

\section{INTRODUCTION}

Environmental reward-predictive stimuli provide a major source of motivation for adaptive reward-seeking behaviors (Corbit and Balleine, 2015). But this incentive motivational value can become amplified in disordered states, allowing cues to become potent triggers for maladaptive behavior such as compulsive overeating (Johnson, 2013) or drug seeking (Robinson et al, 2013). Considerable evidence has implicated nucleus accumbens core (NAc) dopamine signaling in the expression of a cue's motivational value (Berridge, 2007). Modulatory mechanisms in striatal terminals can, however, regulate dopamine release. Of these, the acetylcholine receptor system has emerged as a key player (Cachope and Cheer, 2014; Cragg, 2006; Sulzer et al, 2016). Yet, little is known about the causal role of the NAc cholinergic system in motivated behavior and there is currently no evidence of acetylcholine receptor modulation of naturally-evoked and behaviorally-relevant dopamine.

Acetylcholine receptors are broadly distributed throughout the NAc and consist of two major subtypes: muscarinic and nicotinic. Tonically-active, cholinergic interneurons provide the primary source of acetylcholine acting at these receptors (Bolam et al, 1984). Nicotinic acetylcholine receptors

* Correspondence: Dr KM Wassum, Department of Psychology, UCLA, I 285 Franz Hall, Box 95I563, Los Angeles, CA 90095-I563, USA, Tel: 3108255443 , Fax: 310206 5895, E-mail: kwassum@ucla.edu

Received I March 2016; revised 24 May 2016; accepted 25 May 2016; accepted article preview online 31 May 2016
(nAChR) on striatal dopamine terminals (Jones et al, 2001) are known to be particularly relevant to the regulation of striatal dopamine signaling (Cachope et al, 2012; Exley et al, 2008a; Threlfell et al, 2012). Muscarinic acetylcholine receptor (mAChR) activity also contributes to dopamine modulation both via regulation of endogenous activity at nAChRs on dopamine terminals (Threlfell et al, 2010) and through activation of $\mathrm{mAChRs}$ on dopamine terminals (Foster et al, 2014; Shin et al, 2015).

Striatal cholinergic interneuron activity correlates with the presentation of reward-predictive stimuli and with motivated behavior (Morris et al, 2004; Nougaret and Ravel, 2015; Ravel et al, 2003). NAc mAChRs have been implicated in instrumental activity (Ikemoto et al, 1998; Pratt and Kelley, 2004), but neither their precise contribution, nor involvement in cue-motivated behavior has been determined. Therefore, here we tested the hypothesis that NAc endogenous $\mathrm{mAChR}$ and $\mathrm{nAChR}$ activity regulates expression of the motivational value of reward-predictive cues and then examined a potential dopaminergic mechanism for this influence.

To achieve this, we first evaluated the influence of NAc mAChR or nAChR blockade on cue-motivated behavior using the Pavlovian-to-instrumental transfer (PIT) task. This task assesses the motivating influence of a reward-predictive cue over the performance of an independently-trained, instrumental reward-seeking action, and thereby isolates the incentive motivational value of the cue from other processes through which cues trigger action, such as their 
ability to exert discriminative control over performance through stimulus-response learning. In a second experiment, we examined the impact of $\mathrm{nAChR}$ or $\mathrm{mAChR}$ blockade on cue-evoked dopamine signaling in the NAc during PIT.

\section{MATERIALS AND METHODS}

\section{Subjects}

All procedures were conducted in accordance with the National Research Council's Guide for the Care and Use of Laboratory Animals and were approved by the UCLA Institutional Animal Care and Use Committee. Male rats served as the subjects for these experiments (Experiment 1: final $n=24$, five rats were excluded due to the misplaced cannula, three due to illness; Long Evans; 300-330 g; Charles River Laboratories, Wilmington, MA; Experiment 2: final $n=10$, an additional five rats were run through the behavioral task, but were excluded from the electrochemical analysis due to misplaced electrodes $(n=2)$, or noisy electrodes and/or an inability to reliably record a significant unexpected food-pelleted-evoked dopamine response $(n=3)$; Sprague Dawley; 280-320 g). Rats were group-housed and handled for 5-7 days prior to the onset of the experiment. Experimentation took place during the dark phase of the $12: 12 \mathrm{~h}$ reverse dark:light cycle. Rats were food deprived receiving $12-14 \mathrm{~g} /$ day of their maintenance diet to maintain $85-90 \%$ free-feeding body weight. Free access to tap water was provided in the home cage.

\section{Surgical Procedures}

In Experiment 1, rats were bilaterally implanted with 22-gauge, stainless steel, guide cannulae (Plastics One, Roanoke, VA) targeted $1 \mathrm{~mm}$ above the intended NAc core infusion site (coordinates relative to Bregma: AP+1.3 mm, $\mathrm{ML} \pm 1.8$, and DV -6.2). Coordinates were selected based on the previous findings demonstrating effects of cholinergic receptor antagonists on reward-related behavior (Nadal et al, 1998; Pratt and Kelley, 2004) and to avoid spread into the NAc shell. For Experiment 2, calibrated carbon-fiber microelectrodes coupled with guide cannulae were implanted unilaterally into the NAc (AP: $+1.3 \mathrm{~mm}$; ML: $\pm 1.3 ; \mathrm{V}:-7.0$ from dura) along with an $\mathrm{Ag} / \mathrm{AgCl}$ reference electrode in the contralateral cortex. Coordinates were selected to match our previous NAc core dopamine recording location during PIT (Aitken et al, 2016; Ostlund et al, 2014b; Wassum et al, 2013) and to avoid electrode place in the anterior commissure. Electrode/cannula probes were arranged such that the electrode tip was aligned to the coordinate above, with the cannula positioned more laterally parallel to the coronal suture. Standard aseptic stereotaxic procedures were used as described previously (Aitken et al, 2016). Following surgery, rats were individually housed and allowed to recover for 5-7 days. Placement(s) were verified using the standard histological procedures described previously (Collins et al, 2016); (see Supplementary Figures 1 and 2).

\section{Behavioral Task}

The PIT behavioral task procedures are similar to those described previously (Wassum et al, 2011, 2013). For
Experiment 1, training and testing took place in a set of 16 Med Associates (East Fairfield, VT) operant chambers described previously (Malvaez et al, 2015). For Experiment 2, training and testing were conducted in a set of two chambers identical to the training chambers, but outfitted with a voltammetric recording unit as described previously (Aitken et al, 2016; Collins et al, 2016).

Pavlovian conditioning. Rats first received 8 days of Pavlovian training in which one of two auditory cues (75 dB tone or white noise; counterbalanced across rats; $\mathrm{CS}^{+}$) was paired with noncontingent delivery of $45-\mathrm{mg}$ grain pellets (Bio-Serv, Frenchtown, NJ). Each $\mathrm{CS}^{+}$was presented for $2 \mathrm{~min}$, during which pellets were presented on a random-time 30 -s schedule. The $\mathrm{CS}^{+}$was presented $6 \mathrm{x} /$ session with a random $2-4 \mathrm{~min}$ intertrial interval (mean $=3 \mathrm{~min}$ ). The lever was never present during these sessions.

Instrumental conditioning. All rats then received 8 days of instrumental training in which lever pressing was rewarded with delivery of a single grain pellet. Each session lasted until 20 outcomes had been earned or 30 min elapsed. Rats received 1 day each of continuous, random-interval $15 \mathrm{~s}$, and randominterval $30 \mathrm{~s}$ schedules of reinforcement, followed by 5 days on the final random-interval $60 \mathrm{~s}$ schedule. The $\mathrm{CS}^{+}$was never present during this training.

Retraining and $C S^{\varnothing}$ habituation. Following instrumental training, rats in Experiment 1 underwent surgery and, after recovery, were given two instrumental retraining sessions and one Pavlovian retraining session. Rats in Experiment 2 received one Pavlovian $\mathrm{CS}^{+}$reminder session after instrumental training. Following this, both groups of rats received one session of habituation to the neutral control stimulus $\left(C S^{\varnothing}\right)$, which consisted of 6,2 -min presentations of the $C S^{\varnothing}$ (opposite stimulus as the $\mathrm{CS}^{+}$), with a 2-4 min intertrial interval. No rewards were delivered during this session. Rats were also given 2 days of instrumental and 1 day of Pavlovian retraining in between each PIT test.

PIT test. Rats were given three total PIT tests to allow a within-subjects drug-treatment design. On the day prior to each PIT test, rats in Experiment 1 were given a single 30min instrumental extinction session in which no cues were present, and lever pressing was unrewarded. During each PIT test, the lever was continuously available, but pressing was not reinforced. Responding was extinguished for $5 \mathrm{~min}$ to establish a low rate of baseline performance, after which each CS was presented four times in pseudorandom order, also without accompanying reward. Each CS lasted 2 min with a 4-min fixed intertrial interval. PIT has been robustly demonstrated in both Long Evans (Corbit et al, 2007; Wassum et al, 2011) and Sprague Dawley (Saddoris et al, 2011; Wassum et al, 2013) rats with procedures similar to those here, and the PIT effect was shown here to be similar in magnitude in the control conditions in both species.

\section{Drugs and Infusion Procedures}

In Experiment 1, immediately prior to each PIT test, rats were bilaterally infused with either the selective nAChR 
antagonist mecamylamine $(10 \mu \mathrm{g} /$ side; Tocris Bioscience, Bristol, UK), the selective $\mathrm{mAChR}$ antagonist scopolamine (10 $\mu \mathrm{g} /$ side; Tocris), or an equivalent volume of sterile artificial cerebral spinal fluid (ACSF; Tocris). All rats were tested under each drug condition, counterbalanced for order. Doses were selected based on their ability to alter reward seeking when infused into the NAc, without causing gross motor impairments (Nadal et al, 1998; Nunes et al, 2013; Pratt and Kelley, 2004). Drugs were infused, as described previously (Wassum et al, 2009), into the NAc in a volume of $0.5 \mu$ l over $1 \mathrm{~min}$ via an injector inserted into the guide cannula fabricated to protrude $1 \mathrm{~mm}$ ventral to the cannula tip, using a microinfusion pump. For Experiment 2, rats were unilaterally infused with either mecamylamine $(10 \mu \mathrm{g} / \mathrm{side})$, scopolamine $(10 \mu \mathrm{g} / \mathrm{side})$, or an equivalent volume $(0.5 \mu \mathrm{l} /$ side) of ACSF via the cannula attached to the carbon-fiber microelectrode.

\section{Fast-Scan Cyclic Voltammetry}

For Experiment 2, chronically-implanted and carbon-fiber microelectrodes were used to make longitudinal, withinsubject measures of dopamine concentration changes across multiple tests. These electrodes were coupled to the guide cannula, as described below, to allow infusion of pharmacological agents into the extracellular space surrounding the recording electrode. For each test session, rats were placed in the operant chamber and tethered to the voltammetric recording unit through an electrical swivel. Fast-scan cyclic voltammetry (FSCV) recordings were performed during the PIT tests as described previously (Aitken et al, 2016; Collins et al, 2016). On each test day, after stabilization of the baseline current $(\sim 20 \mathrm{~min})$, rats were unilaterally infused as described above and the baseline was allowed to restabilize for 5-10 min, after which the PIT test commenced with the onset of the house light and insertion of the lever. Because bilateral drug treatment would produce a behavioral effect that would confound our neurochemical analysis (eg, a drug-induced change in the cue-evoked dopamine response could be a secondary consequence of the behavioral effects of that treatment), we used unilateral infusion to minimize such effects. For a subset of rats $(n=7)$, FSCV measurements were also collected prior to, and after the drug infusion and PIT test during the unexpected delivery of five chocolate-flavored 45-mg pellets (Bio-Serv). Behavioral performance did not differ between the subjects that received and those that did not receive food pellets prior to the test (main effect of Pellet exposure group: $F_{2,12}=0.35, p=0.564$ on lever presses during the PIT test; pellet exposure $\times$ drug: $F_{2,12}=0.55$, $p=0.59$; pellet exposure $\times$ CS: $F_{2,12}=0.42, p=0.67$ ).

\section{Carbon-Fiber Microelectrode/Cannula Assembly}

Carbon-fiber microelectrodes were prepared and calibrated (average calibration factor $=45.45 \mathrm{nM} / \mathrm{nA}, \mathrm{SEM}=4.13$ ) as described previously (Clark et al, 2010; Collins et al, 2016; Wassum et al, 2013), with the exception that the silica shaft encasing the carbon fiber was cut to $\sim 12 \mathrm{~mm}$. Following calibration, each electrode was affixed to a 22-gauge, stainless steel guide cannula (Plastics One) with the cannula extending 3-4 $\mathrm{mm}$ beyond the $14-\mathrm{mm}$ plastic threaded base. Injectors (Plastics One) were designed to fit these cannulae and to protrude an additional $3-4 \mathrm{~mm}$. The cannula terminated 3-4 $\mathrm{mm}$ above the tip of the electrode to prevent damage to the recording zone. The lower $\sim 2 / 3$ of the plastic base was shaved down on one side of the cannula to allow a flat space for the electrode to be attached (Figure 1a and b). The electrode was affixed to the guide cannula by two-component clear epoxy (Devcon, Danvers, MA). Using a microscope and microruler, while the epoxy was still loose the electrode position was adjusted along the shaft of the cannula such that the electrode tip was within $100-300 \mu \mathrm{m}$ from the tip of the injector when inserted through the cannula (Figure 1b). Guide cannulae were at an $\sim 3-5^{\circ}$-angle relative to the electrode.

\section{Carbon-Fiber Microelectrode/Cannula Characterization}

In vitro characterization. We calibrated in vitro a sample $(n=8)$ of carbon-fiber microelectrodes either alone or coupled with the cannula and injector (electrode/cannula probes) to determine whether assembly with the cannula and injector placement near electrode tip would disrupt FSCV dopamine detection. Both electrode types showed similar responses to dopamine passed by the electrode at known concentrations of $0.25,0.5$, and $1.0 \mu \mathrm{M}$ (main effect of electrode type: $F_{1,7}=0.78, p=0.41$; dopamine concentration: $F_{3,21}=112.0, p<0.0001$; electrode type $\times$ concentration interaction: $F_{3,21}=0.67, p=0.58$; Figure $1 c$ ).

In vivo characterization. We compared the dopamine response to unexpected food reward delivery detected at the electrode/cannula probes used in the current study (the $n=7$ for which these measures were collected) to that detected at a sample $(n=7)$ of single carbon-fiber microelectrodes from our previous studies and found no difference (average unexpected reward dopamine response electrode only: $34.12 \mathrm{nM}, \mathrm{SEM}=4.04$; electrode/cannula probe: $31.71,3.31$; $t_{12}=0.46, p=0.66 ;$ Figure 1d). Similarly, there was no difference in the dopamine response to an unexpected food reward at electrode/cannula probes prior to $v s$ immediately following an infusion of ACSF (average post-vehicle response: $27.43,2.35 ; t_{12}=1.44, p=0.20$; Figure $1 \mathrm{~d}$ ). We additionally examined whether infusions in the extracellular recording space would elicit any prolonged change in FSCV dopamine detection by looking at the dopamine response to unexpected food reward delivery before any infusion had ever been made compared with the dopamine response after rats had received (on previous days) one or two infusions. In this case, we used the pretest/infusion dopamine response for each of the three tests. We detected no significant difference in reward-evoked dopamine release before any infusion relative to after one or two infusions (average dopamine response, no infusion: $36.63 \mathrm{nM}, \mathrm{SEM}=5.50$; after one infusion: $30.58,3.41$; after two infusions: 31.54 , 3.73; main effect of test: $F_{2,6}=0.81, p=0.41$; Figure 1 e).

Combined, these data show that arrangement of a carbon-fiber microelectrode with a guide cannula and infusion of liquid into the extracellular space surrounding the electrode does not disrupt FSCV dopamine detection or sensitivity. 


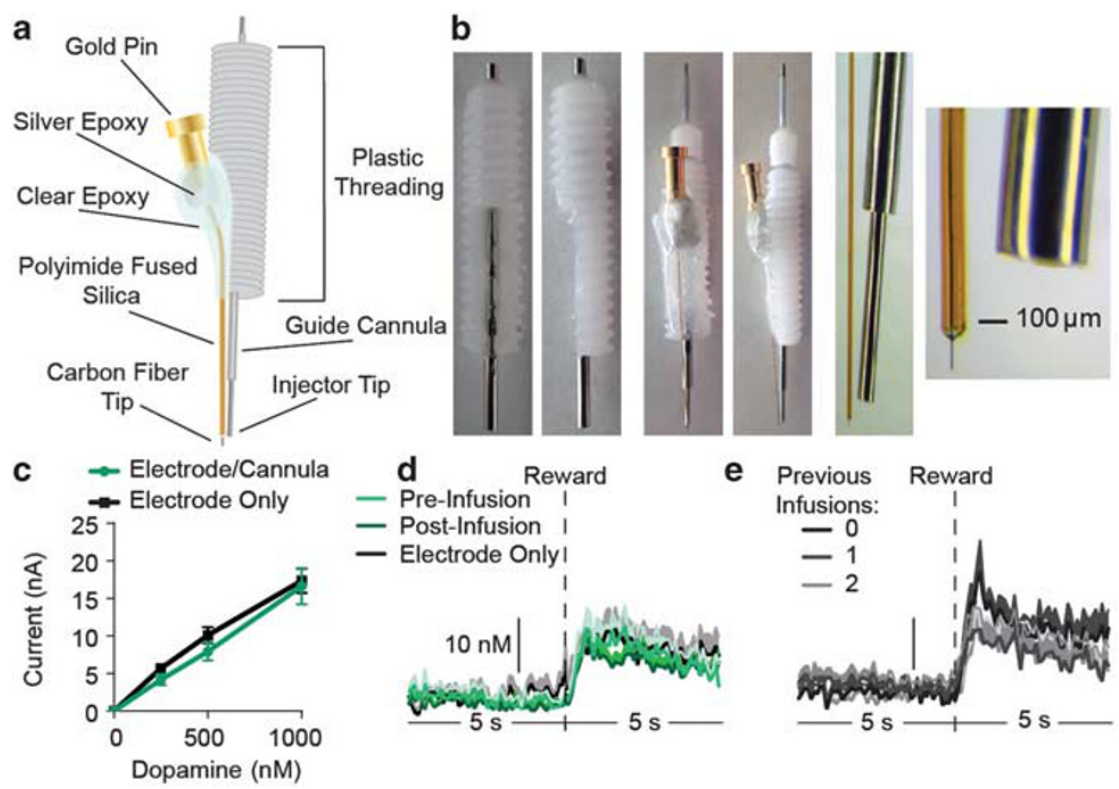

Figure I Design and characterization of carbon-fiber microelectrode/cannula probes. (a) Schematic of carbon-fiber microelectrode combined with a guide cannula for microinfusion in the extracellular recording space. (b) Images of guide cannulae with portion of plastic threading shaved down on one side (left) and assembled electrode/cannula probe with injector inserted through the guide cannula (middle). Right-most images show magnification of the electrode tip with the injector inserted through the guide cannula. (c) Average current response of either electrode/cannula probes or individual electrodes to known concentrations of dopamine in vitro in a flow-cell calibration unit. (d) Averaged (across trials and across subjects) dopamine concentration vs time traces $5 \mathrm{~s}$ before and after delivery of an unexpected food-pellet reward detected at either carbon-fiber microelectrodes alone, or electrode/cannula probes either before (pre-infusion) or after (post-infusion) infusion of ACSF vehicle. (e) Averaged dopamine concentration vs time around pellet delivery detected at electrode/cannula probes either before any infusion had ever been made or after rats had, on previous days, received either one or two infusions.

\section{Data Analysis}

Statistical analysis. Electrochemical data were analyzed using software written in LabVIEW (National Instruments). Data were processed with Microsoft Excel (Redmond, WA). Statistical analyses were conducted with GraphPad Prism (La Jolla, CA) and SPSS (IBM, Chicago, IL). For all hypothesis tests, the $\alpha$ level for significance was set to $p<0.05$. Data were analyzed with one- and two-way ANOVAs (Geisser-Greenhouse correction), paired $t$-tests, correlation, and regression, where appropriate. Bonferroni corrected post-hoc comparisons and planned paired $t$-tests were used to clarify main effects and interactions.

Behavioral analysis. Lever pressing and entries into the food-delivery port were the primary behavioral output measures. During the PIT tests, these measures were counted and averaged across trials for each 2-min CS period, with behavioral output during the 2 -min periods prior to each CS serving as the baseline for CS-induced changes in behavior.

Voltammetric analysis. Principal component regression, a chemometric technique that combines principal component analysis with inverse least-squares regression (Heien et al, 2005), was used to isolate changes in current due to dopamine and $\mathrm{pH}$ from the cyclic voltammetric data. We used a standard training set of dopamine, $\mathrm{pH}$, and drift CVs as has been described previously (Aitken et al, 2016; Collins et al, 2016). This procedure allowed us to distinguish changes in current due to dopamine fluctuations from changes due to $\mathrm{pH}$ or to other electroactive substances. Individual values of residual extraneous variance in an unknown measurement $(Q)$ from the principal component regression analysis were compared with the average tolerance level $(Q \alpha)$, which was 81.026 Q/nA2. On occasion, isolated transient electrical artifacts were detected, as identified by transient $Q$ values well in excess of the $Q \alpha$ in isolated cells. Estimates of dopamine at these time points were interpolated by taking the average of the surrounding cells. Trials in which $>10 \%$ of the data points had residual values in excess of the $Q \alpha$ were omitted from the analysis. This occurred on $17.5 \%$ of all trials, which were evenly distributed between CS and drug conditions. After this analysis, all data were converted to estimated dopamine concentration via an electrode-specific, in vitro, pretest calibration factor.

Voltammetric data were isolated starting $30 \mathrm{~s}$ before the onset of the CS and ending with the offset of the 120-s CS. For each trial, the average of current across the 30-s period prior to CS onset served as the baseline for background subtraction. Maximal (ie, peak) dopamine concentration change during the first $30 \mathrm{~s}$ following CS onset provided a quantification of the CS onset-induced dopamine response. This has been previously demonstrated as a reliable measure of task-related and cue-evoked dopamine signaling (Aitken et al, 2016; Ostlund et al, 2014b; Wassum et al, 2013). Because previous data have demonstrated $\mathrm{CS}^{+}$-induced elevations in NAc dopamine signaling during PIT (Aitken et al, 2016; Ostlund et al, 2014b; Wassum et al, 2013), planned comparisons were used when comparing peak dopamine concentration during the $\mathrm{CS}^{+} v s \mathrm{CS}^{\varnothing}$. The average of the background-subtracted dopamine concentration change during the entire 2-min CS period served as a 


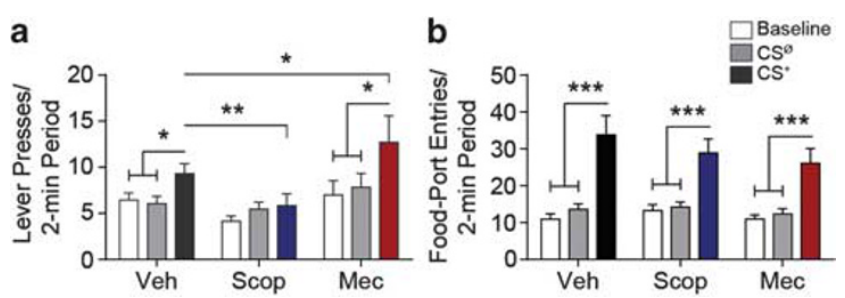

Figure 2 Effect of nucleus accumbens muscarinic and nicotinic acetylcholine receptor blockade on Pavlovian-to-instrumental transfer. Prior to each PIT test (see the 'Materials and Methods' section), rats were bilaterally infused with either scopolamine (Scop; $10 \mu \mathrm{g}$ ), mecamylamine (Mec; $10 \mu \mathrm{g}$ ), or ACSF vehicle (Veh) into the NAc. (a) The PIT effect. Number of lever presses during each 2-min period, averaged across trials compared between the CS-free (baseline), neutral stimulus $\left(C^{\varnothing}\right)$, and reward-predictive cue $\left(\mathrm{CS}^{+}\right)$periods. (b) Conditioned food-port approach responding. Number of entries into the food-delivery port during each 2-min period, averaged across trials compared between the baseline, $C S^{\varnothing}$, and $C S^{+}$periods. $n=24$. Error bars represent \pm I SEM. ${ }^{*} p<0.05$, *** $p<0.0$ I, ***** $p<0.00$ I.

measure of more prolonged CS-induced dopamine concentration changes. For both measures, dopamine concentration change estimates were averaged across the trials of the same type for each rat.

For the unexpected reward-evoked dopamine response, voltametric data were isolated for the $5 \mathrm{~s}$ before and after pellet delivery. For each trial, the average of current across the 5-s period prior pellet delivery served as the baseline for background subtraction, with peak dopamine concentration change in the $3 \mathrm{~s}$ following pellet delivery providing quantification of the response. All subjects collected each pellet within $<3 \mathrm{~s}$ following the delivery.

\section{RESULTS}

For Experiment 1, Pavlovian training was used to pair a 2 -min auditory $\mathrm{CS}^{+}$with food reward delivery. An alternate 2-min auditory stimulus was presented unpaired with reward and served as the control CS . Rats were then instrumentally conditioned, in the absence of the stimuli, to lever press to earn food rewards (see Supplementary Results for training data). In the critical PIT test phase, the lever was available, though pressing was not reinforced, and each CS was presented in pseudorandom order, also without accompanying reward, to assess the motivating influence of the $\mathrm{CS}^{+}$over lever-pressing activity. Changes in lever-press rate during the $\mathrm{CS}^{+}$provided the measure of cue-motivated behavior (ie, expression of PIT). $\mathrm{CS}^{+}$-induced changes in the rate of entry into the food-delivery port were used as a measure of Pavlovian-conditioned goal-approach responses. Rats were given three PIT tests, one each following bilateral intra-NAc infusion of the nonspecific $\mathrm{mAChR}$ antagonist scopolamine, the nonspecific nAChR antagonist mecamylamine, or vehicle.

As can be seen in Figure 2a, blockade of NAc mAChRs and nAChRs differentially impacted motivated behavior (main effect intra-NAc drug: $F_{2,46}=3.52, p=0.04$; CS period: $F_{2,46}=9.43, p=0.0004 ; \quad C S \times$ drug: $F_{4,92}=2.40, p=0.056$ ). Demonstrating PIT, the $\mathrm{CS}^{+}$elevated lever pressing relative to both the baseline and $C S^{\varnothing}$ periods under control conditions $(p<0.05)$. Blockade of NAc mAChRs attenuated the expression of this cue-motivated behavior $(p<0.01)$ and prevented PIT $(p>0.05)$, whereas nAChR blockade enhanced the invigorating influence of the $\mathrm{CS}^{+}(p<0.05)$, producing robust PIT $(p<0.001)$. Acetylcholine receptor blockade predominantly influenced $\mathrm{CS}^{+}$-invigorated responding; neither baseline, nor $\mathrm{CS}^{\varnothing}$ lever-press rate were significantly altered by intra-NAc scopolamine or mecamylamine treatment $(p>0.05)$. In two separate experiments, similar effects were produced at multiple drug doses (Supplementary Figure 3).

The effects of both intra-NAc scopolamine and mecamylamine were limited to the motivating influence of the $\mathrm{CS}^{+}$ over instrumental behavior. Intra-NAc infusion of neither scopolamine, nor mecamylamine significantly impacted the performance of the Pavlovian-conditioned food-port approach response (main effect of CS period: $F_{2,46}=47.35$, $p<0.0001$; drug: $F_{2,46}=0.55, p=0.58$; CS $\times$ drug: $F_{4,92}=1.06$, $p=0.38$; Figure $2 \mathrm{~b}$ ). In all cases, the $\mathrm{CS}^{+}$induced a robust elevation in food-port entries relative to both the $\mathrm{CS}^{\varnothing}$ and baseline periods $(p<0.001)$.

These data suggest that NAc mAChRs and nAChRs have functionally important, but opposing roles in expression of the motivational value that allows reward-predictive cues to invigorate instrumental activity. Although there are other potential mechanisms for these behavioral effects, they do appear analogous to those demonstrating opposing influences of mAChR and nAChR activity on phasic striatal dopamine release. In the slice, blockade of striatal nAChRs located presynaptically on dopamine terminals (Jones et al, 2001) can modulate the release of dopamine in a manner dependent upon the stimulated firing pattern of dopamine neurons (Zhang and Sulzer, 2004; Zhang et al, 2009). Under some conditions, inactivation of these nAChRs can actually augment the release of dopamine generated by high-frequency stimulation (simulating burst firing) (Rice and Cragg, 2004). Activating $\mathrm{mAChR}$ autoreceptors on striatal cholinergic interneurons, which can decrease acetylcholine tone at nAChRs on dopamine terminals, can also augment terminal dopamine release to high-frequency stimulation (Shin et al, 2015; Threlfell et al, 2010). Not only is it well established that reward-predictive cues trigger burst firing in dopamine cells (Schultz, 2001), but there is growing evidence that the resulting dopamine release in the NAc mediates cue-motivated instrumental behavior (Lex and Hauber, 2008; Ostlund et al, 2014b; Peciña and Berridge, 2013). Combined, these data suggest that blockade of nAChR could enhance the dopamine response to reward-predictive cues, whereas antagonizing $\mathrm{mAChRs}$ could blunt it.

We tested this hypothesis in Experiment 2 by assessing the influence of blockade of either nAChRs or mAChRs on cueevoked NAc dopamine signaling during PIT. To achieve this, we devised a probe consisting of a carbon-fiber microelectrode affixed to a guide cannula (see Figure 1) to allow FSCV dopamine measurement directly following the infusion of $\mathrm{nAChR}$ or mAChR antagonists into the extracellular space surrounding the recording electrode. Rats were unilaterally implanted with these probes in the NAc and trained as described above. These chronically-implanted microelectrode/cannula probes allowed within-subject measurement of cue-evoked dopamine responses during each of three PIT tests: one each following the unilateral infusion of scopolamine, mecamylamine, or vehicle. 


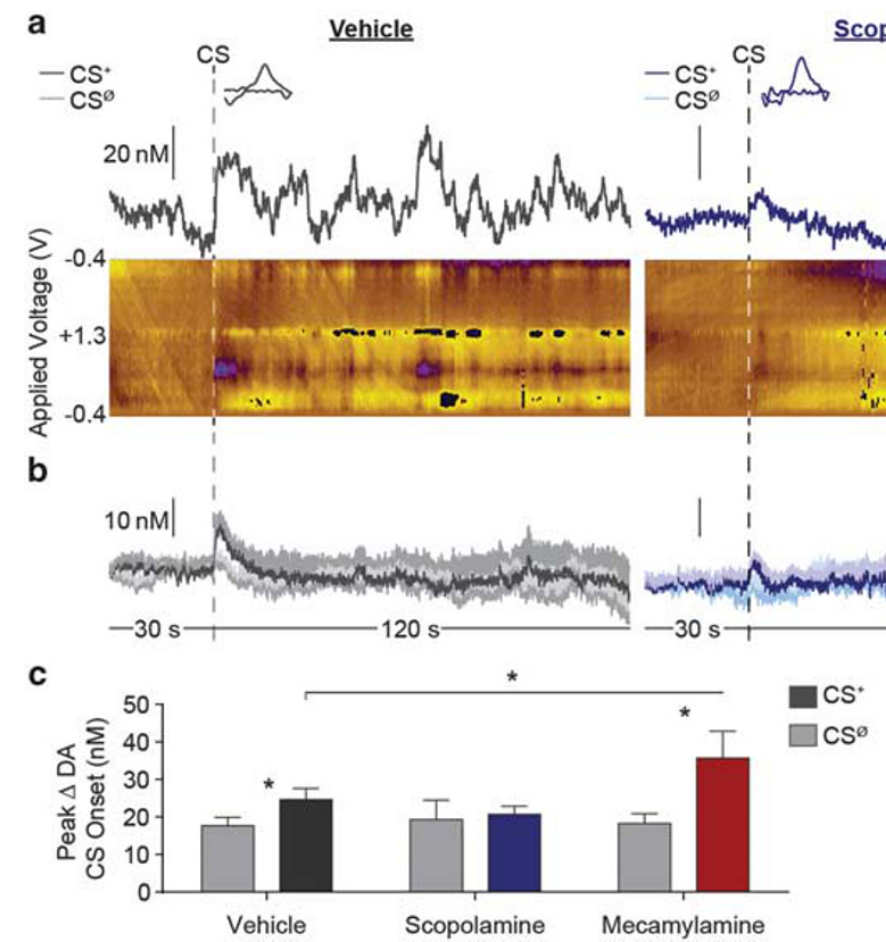

copolamine

Mecamylamine 
a
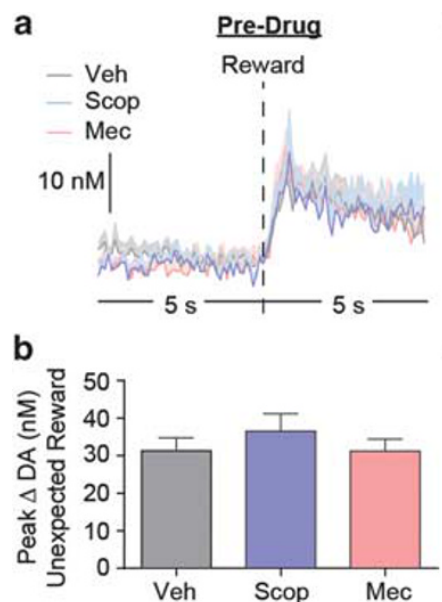

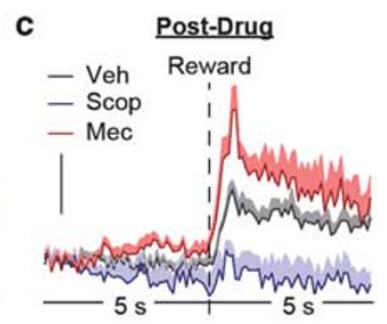

.

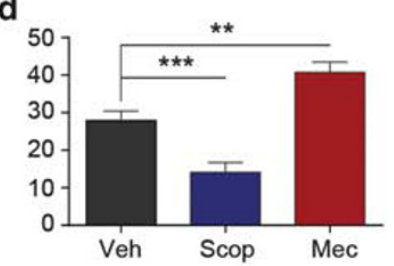

Figure 4 Effect of nucleus accumbens muscarinic and nicotinic acetylcholine receptor blockade on unexpected reward-evoked dopamine. (a and c) Averaged (across trials and across subjects) dopamine concentration vs time traces $5 \mathrm{~s}$ before and after delivery of an unexpected food pellet reward off drug (a) or following the unilateral infusion of either scopolamine (Scop; $10 \mu \mathrm{g})$, mecamylamine (Mec; $10 \mu \mathrm{g}$ ), or ACSF vehicle (Veh) into the NAc FSCV recording zone (c). Shading reflects + I between-subjects SEM. (b and d) Peak dopamine concentration change within $3 \mathrm{~s}$ following unexpected food reward delivery off drug (b) or following unilateral intra-NAc infusion (d) $n=7$. Error bars represent \pm I SEM. *** $p<0.01$, ***** $p<0.001$

\section{DISCUSSION}

Here we evaluated the influence of NAc core nAChR and mAChR blockade on both cue-motivated behavior and cue-evoked dopamine signaling. Blockade of mAChRs attenuated, whereas $\mathrm{nAChR}$ blockade augmented the invigorating influence of a reward-predictive cue over instrumental reward-seeking activity. Providing a potential mechanism for these behavioral effects, disruption of mAChR activity was found to blunt, and nAChR inactivation to augment cue-evoked dopamine release. Similarly, NAc $\mathrm{mAChR}$ and $\mathrm{nAChR}$ inactivation attenuated, or augmented, respectively, the phasic dopamine response to unexpected reward. These data demonstrate cholinergic receptor modulation of naturally-evoked and behaviorally-relevant dopamine signaling in awake-behaving animals and point to the functional significance of cholinergic modulation of NAc dopamine signaling in mediating expression of the motivational value that allows reward-predictive cues to invigorate reward-seeking activity.

Nucleus accumbens core nAChR and mAChRs were found to have opposing roles in cue-motivated behavior. Endogenous activity at NAc nAChRs serves to limit the invigorating influence of cues over instrumental action, as evidenced by the heightened PIT effect observed following $\mathrm{nAChR}$ blockade. The blunted PIT effect observed following $\mathrm{mAChR}$ inactivation suggests endogenous NAc mAChR activity facilitates this expression of the cue's motivational value. Intra-NAc scopolamine infusion recapitulated the suppressive effect of systemic administration of this drug on PIT (Ostlund et al, 2014a), but interestingly, intra-NAc mecamylamine infusion showed an opposite effect to the systemic mecamylamine treatment (PIT enhancement rather than attenuation). This latter finding suggests that nAChRs may

differentially contribute to cue-motivated behavior depending on the brain region. The current results are consistent with previous work generally implicating NAc mAChRs in instrumental activity (Pratt and Kelley, 2004) and extend to show involvement of these receptors in cue-motivated behavior.

The behavioral effects of NAc nAChR and mAChR inactivation were complimented by data showing a corresponding influence of these treatments to enhance or attenuate, respectively, cue onset-evoked NAc dopamine signaling, which has been both correlated with (Aitken et al, 2016; Ostlund et al, 2014b; Wassum et al, 2013) and causally implicated in (Lex and Hauber, 2008; Peciña and Berridge, 2013; Wyvell and Berridge, 2000) the motivating influence of cues over reward-seeking activity. Of note, under control conditions, dopamine was found to be elevated at $\mathrm{CS}^{+}$onset and to return to baseline within $\sim 30 \mathrm{~s}$, similar to our recent report (Aitken et al, 2016), but shorter-lasting than the cueevoked dopamine response detected during PIT in the earlier work (Wassum et al, 2013). This discrepancy likely resulted from one of two methodological differences, the current and Aitken et al. (2016) studies both used a multi-test design and an operant chamber with a recessed food-delivery port, whereas the Wassum et al. (2013) study used a single test and an operant chamber with a protruding food-delivery port that allowed more port visibility and interaction.

There are several potential mechanisms through which blockade of acetylcholine receptor activity could have altered cue-evoked behavior and dopamine release. The effect of scopolamine could have been the result of direct action of this drug on postsynaptic $M_{1}$ mAChRs on NAc medium spiny projection neurons (Goldberg et al, 2012), which could indirectly modulate dopamine signaling through the regulation of midbrain dopamine cell activity. It is also possible that a component of either or both the behavioral and dopamine effects could be indirect via activity at acetylcholine receptors on NAc glutamate terminals. Indeed, cholinergic modulation of striatal dopamine release has been demonstrated, under some conditions, to be sensitive to glutamate receptor antagonists (Cachope et al, 2012; Wonnacott et al, 2000).

Data from ex vivo studies, demonstrating that cholinergic receptor activity can modulate dopamine signaling via direct action at dopamine terminals (Exley et al, 2008a; Exley and Cragg, 2008b; Threlfell et al, 2012), provide a parsimonious mechanism for the results here. Activating presynaptic nAChRs on dopamine terminals with endogenous acetylcholine can trigger dopamine release (Cachope et al, 2012; Threlfell et al, 2012), but ex vivo studies have demonstrated that this modulation depends on the activity level of dopamine cells (Zhang and Sulzer, 2004; Zhang et al, 2009). Nicotinic acetylcholine receptors can facilitate low probability dopamine release, but suppress dopamine release from high-frequency stimulation (mimicking burst firing) (Exley and Cragg, 2008b; Sulzer et al, 2016; Threlfell and Cragg, 2011). Indeed, although disrupting $\mathrm{nAChR}$ signaling can suppress the tonic dopamine measured by microdialysis (Lim et al, 2014), the higher resolution afforded by FSCV shows that it can actually augment high-frequency phasic release under some conditions (Exley et al, 2008a; Rice and Cragg, 2004), an effect we demonstrate here in awakebehaving animals with dopamine activity generated by 
unexpected reward or reward cue presentation. Interestingly, nAChR agonists can also augment high-frequency phasic dopamine release ex vivo (Rice and Cragg, 2004; Zhang and Sulzer, 2004), but they do so through rapidly desensitizing the nAChRs, mimicking an antagonist-like effect (Lim et al, 2014; Zhang and Sulzer, 2004).

Activity at $\mathrm{M}_{2} / \mathrm{M}_{4} \mathrm{mAChR}$ autoreceptors on NAc cholinergic interneurons (Calabresi et al, 1998) can also regulate terminal dopamine release to high-frequency stimulation by regulating acetylcholine tone at nAChRs on dopamine terminals (Threlfell et al, 2010). Recently however, activity at $\mathrm{M}_{5} \mathrm{mAChRs}$ on dopamine terminals has been shown to enhance striatal dopamine (Shin et al, 2015). The current result, demonstrating a blunting of cue- and rewardevoked dopamine release by nonselectively blocking NAc mAChR activity, could be achieved by either mechanism, and future work is needed to determine precisely how these different classes of NAc mAChRs influence behaviorallyrelevant dopamine signaling.

Striatal cholinergic interneuron activity is controlled by a diverse array of afferent inputs (Lim et al, 2014), and cholinergic modulation of dopamine signaling has been demonstrated ex vivo via activation of thalamostriatal inputs (Threlfell et al, 2012). Cholinergic signaling is elevated in situations, such as satiety (Mark et al, 1992) or disadvantageous high effort or low reward conditions (Nougaret and Ravel, 2015) that discourage vigorous reward seeking. Striatal cholinergic interneurons also express receptors for insulin, a satiety and adiposity signal, and activation of these receptors increases cholinergic activity and modulates NAc dopamine signaling through a nAChR-dependent mechanism (Stouffer et al, 2015). Acetylcholine receptor activity could therefore provide one modulatory mechanism over cue-evoked dopamine signaling to regulate the motivating influence of cues according to their current adaptive value. This motivational influence can become excessive and/or disproportionate with need, and this is thought to contribute to overeating (Johnson, 2013) and maladaptive drug seeking (Robinson et al, 2013). These results therefore have implications for the understanding and treatment of compulsive overeating, addiction, and other disorders marked by maladaptive motivation.

\section{FUNDING AND DISCLOSURE}

This research was supported by a Hellman Foundation Fellowship, a UCLA Faculty Career Development award, grant DA035443 from the NIH to KMW, and grants AG045380, DK098709, and DA029035 from the NIH to SBO. The authors declare no conflict of interest.

\section{ACKNOWLEDGMENTS}

We would like to thank Dr Scott Ng-Evans for his expert hardware and software assistance, and Drs Pamela Kennedy and Alicia Izquierdo for helpful discussions.

\section{REFERENCES}

Aitken TJ, Greenfield VY, Wassum KM (2016). Nucleus accumbens core dopamine signaling tracks the need-based motivational value of food-paired cues. J Neurochem 136: 1026-1036.
Berridge KC (2007). The debate over dopamine's role in reward: the case for incentive salience. Psychopharmacology (Berl) 191: 391-431.

Bolam JP, Wainer BH, Smith AD (1984). Characterization of cholinergic neurons in the rat neostriatum. A combination of choline acetyltransferase immunocytochemistry, golgi-impregnation and electron microscopy. Neuroscience 12: 711-718.

Cachope R, Cheer JF (2014). Local control of striatal dopamine release. Front Behav Neurosci 8: 188.

Cachope R, Mateo Y, Mathur BN, Irving J, Wang HL, Morales M et al (2012). Selective activation of cholinergic interneurons enhances accumbal phasic dopamine release: setting the tone for reward processing. Cell Rep 2: 33-41.

Calabresi P, Centonze D, Pisani A, Sancesario G, North RA, Bernardi G (1998). Muscarinic IPSPs in rat striatal cholinergic interneurones. J Physiol 510 Pt 2 421-427.

Clark JJ, Sandberg SG, Wanat MJ, Gan JO, Horne EA, Hart AS et al (2010). Chronic microsensors for longitudinal, subsecond dopamine detection in behaving animals. Nat Methods 7: 126-129.

Collins AL, Greenfield VY, Bye JK, Linker KE, Wang AS, Wassum KM (2016). Dynamic mesolimbic dopamine signaling during action sequence learning and expectation violation. Sci Rep 6: 20231.

Corbit LH, Balleine BW (2015). Learning and motivational processes contributing to Pavlovian-instrumental transfer and their neural bases: dopamine and beyond. Curr Top Behav Neurosci 27: 259-289.

Corbit LH, Janak PH, Balleine BW (2007). General and outcomespecific forms of Pavlovian-instrumental transfer: the effect of shifts in motivational state and inactivation of the ventral tegmental area. Eur J Neurosci 26: 3141-3149.

Cragg SJ (2006). Meaningful silences: how dopamine listens to the ACh pause. Trends Neurosci 29: 125-131.

Exley R, Clements MA, Hartung H, McIntosh JM, Cragg SJ (2008a). Alpha6-containing nicotinic acetylcholine receptors dominate the nicotine control of dopamine neurotransmission in nucleus accumbens. Neuropsychopharmacology 33: 2158-2166.

Exley R, Cragg SJ (2008b). Presynaptic nicotinic receptors: a dynamic and diverse cholinergic filter of striatal dopamine neurotransmission. Br J Pharmacol 153(Suppl 1): S283-S297.

Foster DJ, Gentry PR, Lizardi-Ortiz JE, Bridges TM, Wood MR, Niswender CM et al (2014). M5 receptor activation produces opposing physiological outcomes in dopamine neurons depending on the receptor's location. J Neurosci 34: 3253-3262.

Goldberg JA, Ding JB, Surmeier DJ (2012). Muscarinic modulation of striatal function and circuitry. Handb Exp Pharmacol 208: 223-241.

Heien ML, Khan AS, Ariansen JL, Cheer JF, Phillips PE, Wassum KM et al (2005). Real-time measurement of dopamine fluctuations after cocaine in the brain of behaving rats. Proc Natl Acad Sci USA 102: 10023-10028.

Ikemoto S, Glazier BS, Murphy JM, McBride WJ (1998). Rats selfadminister carbachol directly into the nucleus accumbens. Physiol Behav 63: 811-814.

Johnson AW (2013). Eating beyond metabolic need: how environmental cues influence feeding behavior. Trends Neurosci 36: 101-109.

Jones IW, Bolam JP, Wonnacott S (2001). Presynaptic localisation of the nicotinic acetylcholine receptor beta2 subunit immunoreactivity in rat nigrostriatal dopaminergic neurones. J Comp Neurol 439: 235-247.

Lex A, Hauber W (2008). Dopamine D1 and D2 receptors in the nucleus accumbens core and shell mediate Pavlovianinstrumental transfer. Learn Mem 15: 483-491.

Lim SA, Kang UJ, McGehee DS (2014). Striatal cholinergic interneuron regulation and circuit effects. Front Synaptic Neurosci 6: 22 .

Malvaez M, Greenfield VY, Wang AS, Yorita AM, Feng L, Linker KE et al (2015). Basolateral amygdala rapid glutamate 
release encodes an outcome-specific representation vital for reward-predictive cues to selectively invigorate reward-seeking actions. Sci Rep 5: 12511.

Mark GP, Rada P, Pothos E, Hoebel BG (1992). Effects of feeding and drinking on acetylcholine release in the nucleus accumbens, striatum, and hippocampus of freely behaving rats. J Neurochem 58: 2269-2274.

Morris G, Arkadir D, Nevet A, Vaadia E, Bergman H (2004). Coincident but distinct messages of midbrain dopamine and striatal tonically active neurons. Neuron 43: 133-143.

Nadal R, Chappell AM, Samson HH (1998). Effects of nicotine and mecamylamine microinjections into the nucleus accumbens on ethanol and sucrose self-administration. Alcohol Clin Exp Res 22: 1190-1198.

Nougaret S, Ravel S (2015). Modulation of tonically active neurons of the monkey striatum by events carrying different force and reward information. J Neurosci 35: 15214-15226.

Nunes EJ, Randall PA, Podurgiel S, Correa M, Salamone JD (2013). Nucleus accumbens neurotransmission and effort-related choice behavior in food motivation: effects of drugs acting on dopamine, adenosine, and muscarinic acetylcholine receptors. Neurosci Biobehav Rev 379 Pt A 2015-2025.

Ostlund SB, Kosheleff AR, Maidment NT (2014a). Differential effects of systemic cholinergic receptor blockade on Pavlovian incentive motivation and goal-directed action selection. Neuropsychopharmacology 39: 1490-1497.

Ostlund SB, LeBlanc KH, Kosheleff AR, Wassum KM, Maidment NT (2014b). Phasic mesolimbic dopamine signaling encodes the facilitation of incentive motivation produced by repeated cocaine exposure. Neuropsychopharmacology 39: 2441-2449.

Peciña S, Berridge KC (2013). Dopamine or opioid stimulation of nucleus accumbens similarly amplify cue-triggered 'wanting' for reward: entire core and medial shell mapped as substrates for PIT enhancement. Eur J Neurosci. 37: 1529-1540.

Pratt WE, Kelley AE (2004). Nucleus accumbens acetylcholine regulates appetitive learning and motivation for food via activation of muscarinic receptors. Behav Neurosci 118: 730-739.

Ravel S, Legallet E, Apicella P (2003). Responses of tonically active neurons in the monkey striatum discriminate between motivationally opposing stimuli. J Neurosci 23: 8489-8497.

Rice ME, Cragg SJ (2004). Nicotine amplifies reward-related dopamine signals in striatum. Nat Neurosci 7: 583-584.

Robinson MJ, Robinson TE, Berridge KC (2013). Incentive salience and the transition to addiction. In: Miller PM (ed). Biological Research on Addiction 2. Vol 2. Academic Press, San Diego, CA, USA, pp 391-399.

Saddoris MP, Stamatakis A, Carelli RM (2011). Neural correlates of Pavlovian-to-instrumental transfer in the nucleus accumbens shell are selectively potentiated following cocaine self-administration. Eur J Neurosci 33: 2274-2287.

Schultz W (2001). Reward signaling by dopamine neurons. Neuroscientist 7: 293-302.

Shin JH, Adrover MF, Wess J, Alvarez VA (2015). Muscarinic regulation of dopamine and glutamate transmission in the nucleus accumbens. Proc Natl Acad Sci USA. 112: 8124-8129.

Stouffer MA, Woods CA, Patel JC, Lee CR, Witkovsky P, Bao L et al (2015). Insulin enhances striatal dopamine release by activating cholinergic interneurons and thereby signals reward. Nat Commun 6: 8543.

Sulzer D, Cragg SJ, Rice ME (2016). Striatal dopamine neurotransmission: regulation of release and uptake. Basal Ganglia 6: 123-148.

Threlfell S, Clements MA, Khodai T, Pienaar IS, Exley R, Wess J et al (2010). Striatal muscarinic receptors promote activity dependence of dopamine transmission via distinct receptor subtypes on cholinergic interneurons in ventral versus dorsal striatum. J Neurosci 30: 3398-3408.

Threlfell S, Cragg SJ (2011). Dopamine signaling in dorsal versus ventral striatum: the dynamic role of cholinergic interneurons. Front Syst Neurosci 5: 11.

Threlfell S, Lalic T, Platt NJ, Jennings KA, Deisseroth K, Cragg SJ (2012). Striatal dopamine release is triggered by synchronized activity in cholinergic interneurons. Neuron 75: 58-64.

Wassum KM, Ostlund SB, Balleine BW, Maidment NT (2011). Differential dependence of Pavlovian incentive motivation and instrumental incentive learning processes on dopamine signaling. Learn Mem 18: 475-483.

Wassum KM, Ostlund SB, Loewinger GC, Maidment NT (2013). Phasic mesolimbic dopamine release tracks reward seeking during expression of Pavlovian-to-instrumental transfer. Biol Psychiatry 73: 747-755.

Wassum KM, Ostlund SB, Maidment NT, Balleine BW (2009). Distinct opioid circuits determine the palatability and the desirability of rewarding events. Proc Natl Acad Sci USA 106: 12512-12517.

Wonnacott S, Kaiser S, Mogg A, Soliakov L, Jones IW (2000). Presynaptic nicotinic receptors modulating dopamine release in the rat striatum. Eur J Pharmacol 393: 51-58.

Wyvell CL, Berridge KC (2000). Intra-accumbens amphetamine increases the conditioned incentive salience of sucrose reward: enhancement of reward "wanting" without enhanced "liking" or response reinforcement. J Neurosci 20: 8122-8130.

Zhang H, Sulzer D (2004). Frequency-dependent modulation of dopamine release by nicotine. Nat Neurosci 7: 581-582.

Zhang L, Doyon WM, Clark JJ, Phillips PE, Dani JA (2009). Controls of tonic and phasic dopamine transmission in the dorsal and ventral striatum. Mol Pharmacol 76: 396-404.

Supplementary Information accompanies the paper on the Neuropsychopharmacology website (http://www.nature.com/npp) 